\title{
Immunization of bacon pigs against Aujeszky's disease using two different vaccines with oil adjuvants Study of local reactions
}

\author{
P. VANNIER \\ Ministère de l'Agriculture, Station de Pathologie Porcine, \\ B.P. 9, Les Croix, 22440 Ploufragan
}

\begin{abstract}
A systematic inquiry was made at the slaughterhouse to explain the occurrence of inflammatory lesions on the spine of pigs vaccinated with inactivated virus vaccines against Aujeszky's disease. A total of 732 pigs coming from 9 different herds were examined. The effects of two vaccines were compared : the Nobivac Aujeszky (Intervet) and the Geskyvac (Institut Merieux). The lesions were systematically observed and samples were collected for bacteriological and histological studies. In a first trial, 4-6 week-old pigs were vaccinated with both vaccines. In a second one, they were vaccinated at the same age with Nobivac only and in the third one, they were vaccinated with Nobivac at the age of 10 and 16 weeks. The results of the observations showed that the vaccination conditions were not satisfactory as 25 p. 100 of granulomatous lesions were located in the subcutaneous layer. Moreover, in 61 p. 100 of the cases, post-vaccinal primary lesions were modified due to a bacterial superinfection. Lesions induced by the Nobivac vaccine were more constant than those observed after the use of Geskyvac. The last trial showed that the shorter the interval between vaccination and slaughter, the more frequent and the larger the lesions, thus emphasizing the advantage of early piglet vaccination.
\end{abstract}

\section{Immunization of bacon pigs against Aujeszky's disease using live - or inactivated - virus vaccines with or without passive immunity}

\section{P. VANNIER}

\author{
Ministère de l'Agriculture, Station de Pathologie Porcine \\ B.P. 9, Les Croix, 22440 Ploufragan
}

\begin{abstract}
Results of vaccination of pigs, born from immune and non immune sows, with 2 live attenuated viral strains (Alfort 26 and NIA 4) of Aujeszky's disease and one inactivated virus vaccine (Nobivac-Aujeszky) are reported in the present study. Pigs were vaccinated twice by the intranasal and intramuscular routes with the Alfort 26 strain. A third batch of pigs was vaccinated by nebulization of aerosols of the same strain. Pigs vaccinated by the intranasal route were better protected against a challenge exposure made further at the liveweight of $80 \mathrm{~kg}$. Nevertheless, performances of pigs vaccinated by the intramuscular route were quite similar to those of the previous batch. By contrast, vaccination by aerosols did not give the expected results, but the experimental conditions were not optimum. Eightweek-old piglets vaccinated once with the NIA 4 strain by the intranasal route and born from sows immunized with an inactivated virus vaccine (Auskimmune $\mathrm{K}$ ) were well protected against challenge performed 2 months later. The immune response was lower when piglets were vaccinated at the age of 4 weeks in similar conditions. Finally, vaccination by the intramuscular route with an inactivated virus vaccine (Nobivac-Aujeszky) of 4 and 10-weekold piglets born from sows vaccinated four times with the same vaccine induced a good immune response correlated to a mean protection against challenge as compared to pigs of the control group. This last trial done in pigs with a conventional sanitary status cannot be compared with the 2 previous ones. Indeed, a challenge is always less severe in specific pathogen free animals than in pigs with a conventional sanitary status.
\end{abstract}

